PROCEEDINGS OF THE

AMERICAN MATHEMATICAL SOCIETY

Volume 130, Number 6, Pages 1603-1605

S 0002-9939(02)06337-2

Article electronically published on January 21, 2002

\title{
EXPLICIT CONTINUED FRACTIONS WITH EXPECTED PARTIAL QUOTIENT GROWTH
}

\author{
TAKESHI OKANO
}

(Communicated by David E. Rohrlich)

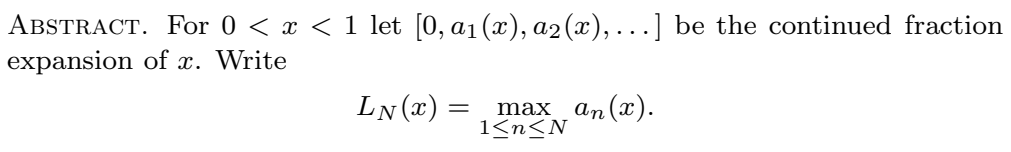

We construct some numbers $x$ 's with

$$
\lim _{N \rightarrow \infty} \inf N^{-1} L_{N}(x) \log \log N=1 / \log 2 .
$$

\section{IntroduCtion}

For $0<x<1$ let $\left[0, a_{1}(x), a_{2}(x), \ldots\right]$ be the continued fraction expansion of $x$. Write

$$
L_{N}(x)=\max _{1 \leq n \leq N} a_{n}(x) .
$$

W. Philipp [1] proved the following theorem: For almost all $x$

$$
\lim _{N \rightarrow \infty} \inf N^{-1} L_{N}(x) \log \log N=1 / \log 2 .
$$

Previously no explicit numbers were known that satisfied

$$
\lim _{N \rightarrow \infty} \inf N^{-1} L_{N}(x) \log \log N=1 / \log 2 .
$$

In this paper we give a method that may be used to construct examples of such numbers.

\section{Main Result}

Theorem. Let $k, K$ and $M_{0}$ be arbitrary fixed positive numbers with $k>1$ and $K \geq 2$. Let $x=\left[0, a_{1}, a_{2}, \ldots\right]$ be an irrational number such that

$$
\max \left\{a_{n} \mid(\log k)^{2} M K^{k^{M}}<n \leq(\log k)^{2}(M+1) K^{k^{M}}\right\}=\left[K^{k^{M+1}}\right]
$$

and

$$
\max \left\{a_{n} \mid(\log k)^{2}(M+1) K^{k^{M}}<n \leq(\log k)^{2}(M+1) K^{k^{M+1}}\right\} \leq\left[K^{k^{M+1}}\right]
$$

Received by the editors January 2, 2001.

2000 Mathematics Subject Classification. Primary 11A55; Secondary 11K50.

Key words and phrases. Continued fractions, measure theory. 
where $M$ is a positive integer with $M>M_{0}$ and $\left[K^{k^{M+1}}\right]$ is the integral part of $K^{k^{M+1}}$. Then

$$
\lim _{N \rightarrow \infty} \inf N^{-1} L_{N}(x) \log \log N=1 / \log k .
$$

Proof. Put $N_{0}=\left[(\log k)^{2} M_{0} K^{k^{M_{0}}}\right]$ and choose $M^{\prime}>M_{0}$ such that $L_{N_{0}}(x) \leq$ $\left[K^{k^{M^{\prime}+1}}\right]$. Let $M$ be any integer greater than $M^{\prime}$. We now consider two cases.

Case 1. $(\log k)^{2} M K^{k^{M}}<N \leq(\log k)^{2}(M+1) K^{k^{M}}$.

Suppose that $M$ is a sufficiently large integer. In this case, we find that $L_{N}(x) \geq$ $\left[K^{k^{M}}\right]$ and

$$
\log \log N=M \log k+O(1) \quad(M \rightarrow \infty)
$$

Hence

$$
N^{-1} L_{N}(x) \log \log N \geq \frac{\left[K^{k^{M}}\right](M \log k+O(1))}{(\log k)^{2}(M+1) K^{k^{M}}} \quad(M \rightarrow \infty) .
$$

Case 2. $(\log k)^{2}(M+1) K^{k^{M}}<N \leq(\log k)^{2}(M+1) K^{k^{M+1}}$.

In this case, we find that $L_{N}(x)=\left[K^{k^{M+1}}\right]$ and

$$
\log \log N=M \log k+O(1) \quad(M \rightarrow \infty) .
$$

Hence

$$
\begin{aligned}
\inf \left\{N^{-1} L_{N}(x) \log \log N \mid(\log k)^{2}(M+1) K^{k^{M}}<N \leq(\log k)^{2}(M+1) K^{k^{M+1}}\right\} \\
=\frac{\left[K^{k^{M+1}}\right](M \log k+O(1))}{(\log k)^{2}(M+1) K^{k^{M+1}}} \quad(M \rightarrow \infty) .
\end{aligned}
$$

Then by these two cases, we can deduce that

$$
\lim _{N \rightarrow \infty} \inf N^{-1} L_{N}(x) \log \log N=1 / \log k .
$$

This completes the proof.

\section{EXAMPLE}

Let $x=\left[0, a_{1}, a_{2}, \ldots\right]$ be an irrational number. Let $K$ and $M$ be as in the Theorem, and let $k=2$ in the Theorem. Define

$$
\mu(M):=\left[(\log 2)^{2} M K^{2^{M}}\right]+1 .
$$

By the Theorem, we define $a_{n}$ as follows:

$$
a_{n}:= \begin{cases}{\left[K^{2^{M+1}}\right]} & \text { if } n=\mu(M) \text { for } M \geq 1, \\ 1 & \text { otherwise. }\end{cases}
$$

Now putting $K=2$, we find that $\mu(1)=2, \mu(2)=16, \mu(3)=369, \mu(4)=$ $125948, \ldots$ Hence, we can construct $x$ as follows:

$$
\begin{aligned}
x & =\left[0, a_{1}, a_{2}, a_{3}, \ldots, a_{15}, a_{16}, a_{17}, \ldots, a_{368}, a_{369}, a_{370}, \ldots, a_{125947}, a_{125948}, a_{125949}, \ldots\right] \\
& =\left[0,1,2^{2^{2}}, 1, \ldots, 1,2^{2^{3}}, 1, \ldots, 1,2^{2^{4}}, 1, \ldots, 1,2^{2^{5}}, 1, \ldots\right] .
\end{aligned}
$$

We can easily see that $x$ is an example of the numbers defined in the Theorem.

\section{ACKNOWLEDGMENTS}

The author thanks the referee for many helpful comments and suggestions. 


\section{REFERENCES}

[1] W. Philipp, A conjecture of Erdös on continued fractions, Acta. Arith. 28 (1976), 379-386. MR 52:8069

Department of Mathematics, Saitama Institute of Technology, Okabe-machi, Saitama 369-0293, JAPAN

E-mail address: okano@sit.ac.jp 